\title{
Micobacteriosis hepato-esplénica, forma inusual de probable tuberculosis extrapulmonar. Caso clínico y revisión bibliográfica
}

\author{
Juan Velásquez M., Muschi Szigethi Q., Rita Panace V., Ricardo Morales I., Silvia Márquez C., \\ Jacqueline Pefaur P., Alfredo Mocarquer M., Pedro Salinas C. y Carlos Beltrán B.
}

\section{Hepatic-splenic micobacteriosis, unusual form of probable extrapulmonary tuberculosis. Case report and review}

We report a 42 years old HIV negative male admitted for fever of unknown origin. Initial laboratory evaluation showed elevated hepatic transaminases and alkaline phosphatase and an hipodense hepatic imagen was visualized in the CT scan. Hepatic biopsy demonstrated tuberculous granulomas and alcohol fast acid rods with Ziehl Neelsen stain. Anti-tuberculous treatment resulted in resolution of fever, improvement of general condition and normalization of laboratory parameters.

Key words: mycobacteriosis, tuberculosis, fever of unknown origin, hepatic granulomatosis.

Palabras claves: micobacteriosis, tuberculosis, fiebre origen desconocido, granulomatosis hepática.

\section{Introducción}

$\mathrm{L}$ a tuberculosis (TBC) es una enfermedad granulomatosa, de etiología infecciosa, causada por Mycobacterium tuberculosis. Su manifestación más habitual es el compromiso pulmonar, mientras que el compromiso extrapulmonar es más frecuente en hospederos inmunocomprometidos ${ }^{1,2}$ y conlleva un desafío diagnóstico importante que requiere una alta sospecha clínica ${ }^{3}$, presentándose muchas veces como un cuadro de fiebre de origen desconocido (FOD).

Histológicamente, la lesión típica es el granuloma caseificante, que se caracteriza por conglomerados formados por células epiteliales, monocitos, macrófagos, fibroblastos y células gigantes multinucleadas, en los que se puede aislar el bacilo de $\mathrm{Koch}^{2}$.

En Chile, desde la década de los 80 se ha visto una reducción progresiva en la prevalencia de la TBC en la población general llegando a tasas inferiores a 20 casos/100.000 habitantes ${ }^{4}$.

Comunicamos el caso de un paciente joven con insuficiencia renal crónica y FOD que resultó ser una micobacteriosis hepato-esplénica.

\section{Caso clínico}

Paciente de sexo masculino, de 42 años de edad, con antecedentes de insuficiencia renal crónica de etiología no precisada, diagnosticada el año 1993, sin control posterior, etilismo crónico y retardo mental leve. Consultó por un cuadro de tres semanas de evolución caracterizado por decaimiento, compromiso del estado general, anorexia, vómitos, dolor abdominal y lumbar, agregándose posteriormente fiebre.

Ingresó febril ( $38^{\circ} \mathrm{C}$ axilar), sin taquicardia, con presión arterial de 161/96 mmHg. Deshidratado, adelgazado y pálido. Presentaba una úlcera malar derecha (diámetro de $2 \mathrm{~cm}$ ), con aumento de volumen, eritema e induración de la zona y secreción purulenta en el ojo derecho. El hígado se proyectaba $2 \mathrm{~cm}$ bajo el reborde costal, sin esplenomegalia. No se palpaban adenopatías.

El laboratorio reveló un hematocrito de $16 \%$, hemoglobina $5,5 \mathrm{~g} / \mathrm{dL}, 8.000$ leucocitos $/ \mathrm{mm}^{3}$ con desviación a izquierda de $5 \%$, VHS $28 \mathrm{~mm} / \mathrm{h}$, PCR: $82 \mathrm{mg} / \mathrm{L}$, creatininemia: $21 \mathrm{mg} / \mathrm{dL}$, nitrógeno ureico: $155 \mathrm{mg} / \mathrm{dL}$, natremia: $135 \mathrm{mEq} / \mathrm{L}$, potasemia: $6 \mathrm{mEq} / \mathrm{L}$, acidosis metabólica compensada, protrombinemia $48 \%$, fosfatasas alcalinas: $232 \mathrm{U} / \mathrm{L}$ (VN: 39-117 U/L), bilirrubinemia: $1,1 \mathrm{mg} / \mathrm{dL}$, gammaglutamil transpeptidasa: $20 \mathrm{U} / \mathrm{L},(\mathrm{VN}$ : 7-32 U/L), transaminasa oxaloacética: $21 \mathrm{U} / \mathrm{L}$ (VN: 10$50 \mathrm{U} / \mathrm{L})$. El cultivo de la úlcera malar fue positivo para Staphylococcus aureus meticilina sensible. Los hemocultivos, urocultivo, serología para VIH (ELISA), HBsAg, VHC (ELISA), VDRL, PPD, baciloscopias de expectoración (dos) y de orina (seis) fueron negativos.

\author{
Hospital Barros Luco Trudeau, \\ Santiago, Chile \\ Residente Servicio de Medicina \\ Interna (JVM) \\ Departamento de Nefrología (RPV, \\ JPP, AMM, PSC). \\ Departamento de Infectología (RMI \\ CBB) \\ Departamento de Gastroenterología \\ (SMC) \\ Universidad de Chile, \\ Facultad de Medicina \\ Estudiante de Medicina (MSQ) \\ Recibido: 10 marzo 2005 \\ Aceptado: 10 agosto 2006
}

Correspondencia a: Juan Velásquez Morales yesterday67@yahoo.com 
Figura 1. Tomografía axial computada de abdomen: hepatomegalia homogénea con imágenes focales hipodensas inespecíficas.

Figura 2. Biopsia hepática: granulomatosis hepática con presencia de bacilo ácido alcohol resistente.

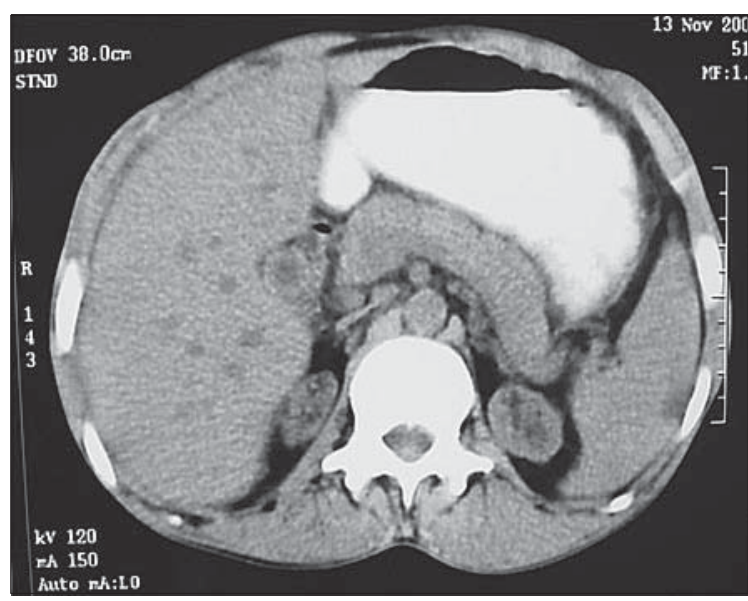

tamiento con fluconazol $150 \mathrm{mg} /$ día oral (dosis ajustada según función renal).

Al décimo día persistía febril por lo que se cambió a anfotericina B deoxicolato $40 \mathrm{mg} / \mathrm{día}(\mathrm{ev})$ junto con iniciar hemodiálisis. Una semana después aún permanecía febril, pesquisándose elevación de parámetros de función hepática: fosfatasas alcalinas de $1.058 \mathrm{U} / \mathrm{L}$, gammaglutamil transpeptidasa de $573 \mathrm{U} / \mathrm{L}$, transaminasa oxalacética de $68 \mathrm{U} / \mathrm{L}$, y valores normales de bilirrubinemia.

Ante la sospecha de candidiasis hepato-esplénica se solicitó TAC de abdomen que mostró hepatomegalia homogénea, con imágenes focales hipodensas inespecíficas aisladas en hígado y bazo, vesícula en porcelana, riñones atróficos e hidroureteronefrosis izquierda (Figura 1). No se visualizaron colecciones, adenopatías ni líquido libre. Ante estos hallazgos y nula respuesta a anfotericina B (700 $\mathrm{mg}$ totales), se decidió efectuar una biopsia hepática transparietal cuyo estudio histológico reveló la existencia de granulomatosis hepática con presencia de bacilos alcoholacido resistentes (BAAR) (Figura 2); la realización de cultivo no fue factible ya que la muestra obtenida fue escasa.

Con la hipótesis de tratarse de una TBC, se efectuó la terapia específica con isoniazida, pirazinamida, etambutol y rifampicina, ajustando los fármacos según el peso y función renal del paciente, hasta completar 7 meses. La curva febril cayó al segundo día de tratamiento, con una mejoría concomitante del estado general y del laboratorio hepático. Al momento de este reporte el paciente está asintomático, permanece en hemodiálisis y completó terapia anti-tuberculosa.

La radiografía de tórax fue normal y la ecografía abdominal demostró evidencias de daño hepático crónico moderado, hepato-esplenomegalia, bazo de 13 $\mathrm{cm}$, colelitiasis y riñones pequeños simétricos. Sin ascitis.

Se realizó hidratación parenteral, corrección del estado ácido-base y electrolítico, con lo cual se evidenció una mejoría importante de los parámetros nitrogenados y se indicó terapia antimicrobiana empírica con ceftriaxona $1 \mathrm{~g} /$ día (ev) y cloxacilina $2 \mathrm{~g} \mathrm{c} / 6 \mathrm{~h}(\mathrm{ev})$ durante diez días, con mejoría significativa de la úlcera malar.

Evolucionó con persistencia de la fiebre asociándose diarrea sin elementos patológicos; un coprocultivo demostró abundante desarrollo de Candida albicans. A esto se agregó progresiva leucopenia llegando a 2.900 leucocitos por lo que se decidió realizar biopsia medular que fue informada como: "hiperplasia eosinofílica" y el mielocultivo arrojó la presencia de Candida albicans. Con estos elementos se planteó el diagnóstico de candidiasis diseminada y se inició tra-

\section{Discusión}

La TBC, enfermedad que en décadas pasadas parecía controlada, en especial en países desarrollados, cobra actualmente mayor fuerza por distintos factores tales como migraciones poblacionales, enfermedades ${ }^{2-7}$, y tratamientos que conllevan inmunosupresión ${ }^{6,8}$, además de pesquisa insuficiente 9 , por lo que, últimamente se ha observado en el mundo un aumento de casos tanto en su forma clásica como atípica ${ }^{10,11}$.

Nuestro país no escapa a esta realidad y, si bien se describe un descenso sostenido de la morbilidad entre los años 1990 y 2000, ésta no ha disminuido en la misma proporción en los recientes ${ }^{4}$.

La fiebre de origen desconocido puede ser una forma de presentación de la TBC, en especial si ésta es de origen extrapulmonar. En esta última destacan además síntomas sistémicos inespecíficos como baja de peso, diaforesis nocturna, astenia, anorexia, adinamia y otros específicos según sea el órgano afectado ${ }^{1,12}$. 


\section{Caso clínico}

En los últimos años la frecuencia de TBC extrapulmonar se ha incrementado por diversos factores tales como: la mejoría en el diagnóstico, mayor número de pacientes inmunocomprometidos (infección por VIH) y otras condiciones ${ }^{13}$.

$\mathrm{Su}$ mecanismo patogénico puede ser por reactivación de focos de bacilemia primaria, propagación de TBC pulmonar o TBC primaria extrapulmonar ${ }^{2}$. El principal mecanismo de diseminación es el hematógeno ${ }^{14}$.

Al involucrar sitios relativamente inaccesibles, requiere una alta sospecha clínica, por lo que el diagnóstico y tratamiento oportuno la mayoría de las veces es tardío, haciéndose muchas veces necesario el uso de procedimientos invasores, con confirmación bacteriológica más difícil ${ }^{13}$.

En la pesquisa son útiles los antecedentes de contacto epidemiológico, TBC previa, presencia de infección por VIH/SIDA u otro tipo de inmunodepresión ${ }^{2}$.

Se describe afección hepática con frecuencia variable en todas las presentaciones de $\mathrm{TBC}^{2}$, pero el compromiso hepático exclusivo es inusual y generalmente asintomático ${ }^{6,8,12,14,15}$, más aún en pacientes inmunocompetentes, por lo que puede ser un hallazgo en biopsias y necropsias ${ }^{2}$.

La forma aislada de TBC hepática puede presentarse como FOD, pero también con síntomas como hepatomegalia dolorosa, (hasta $80 \%$ ) y esplenomegalia $(20 \text { a } 40 \%)^{6,12,14}$, características que presentaba nuestro paciente, o simular patología biliar o tumoral, con ascitis e ictericia, como manifestaciones inusuales ${ }^{6,8,14}$.

Se puede asociar con foco pulmonar o gastrointestinal y rara vez con insuficiencia hepática ${ }^{6,16}$. Las alteraciones al laboratorio son inespecíficas destacando la existencia de anemia normocítica-normocroma, monocitosis, VHS elevada y PPD positivo ${ }^{4}$. Sin embargo, mucho más orientador es la elevación de fosfatasas alcalinas y transaminasas, en especial la GGT sin elevación importante de $\mathrm{GOT}^{1,14}$, perfil encontrado en nuestro caso.

Dentro de los exámenes imagenológicos, la radiografía de tórax suele ser normal ${ }^{8}$ y la ecotomografía abdominal aunque inespecífica, puede mostrar lesiones micronodulares hipodensas, calcificaciones miliares o lesiones con calcificaciones centrales ${ }^{8,12}$.

La TAC es la prueba de mayor sensibilidad y especificidad diagnóstica, permitiendo visualizar lesiones micronodulares únicas y/o múltiples hipodensas o lesiones macronodulares, estas últimas más $\operatorname{raras}^{12,14,17}$.

La sospecha diagnóstica requiere confirmación histológica, mediante la laparoscopia y biopsia hepática $^{14,16}$ (Tabla 1).

La presencia de granulomas caseificantes, BAAR y cultivo positivo son considerados patognomónicos de micobacteriosis, pero estos hallazgos son poco habituales, por lo que su ausencia no descarta compromiso hepático, en especial en áreas endémicas ${ }^{6,8,12,18}$.

La localización más frecuente de los granulomas tuberculoides hepáticos es lobulillar. Se disponen habitualmente en forma micronodular o miliar y en raras ocasiones en forma macronodular o seudotumoral. Por esto, en los diagnósticos diferenciales de hepatitis granulomatosa se deben considerar sarcoidosis, metástasis, infección fúngica y linfoma ${ }^{1,6,17}$.

En nuestro paciente, si bien no se pudo documentar la etiología tuberculosa del compromiso hepático, la favorable y pronta respuesta a la terapia anti-TBC nos permitieron concluir que se tratara de una hepatitis granulomatosa de origen tuberculoso.

El cultivo microbiológico en medio específico (Lowenstein Jenssen) es el estándar de oro para el diagnóstico de $\mathrm{TBC}$, desgraciadamente no son infrecuentes las situaciones clínicas en que no se dispone de una muestra oportuna para el cultivo, pues no se sospecha el diagnóstico al momento de efectuar biopsia de los tejidos; en estas circunstancias, se ha destacado la utilidad de la reacción de polimerasa en cadena (RPC) que posee una alta sensibilidad y permiten distinguir la TBC de otras micobacteriosis ${ }^{8}$.

\section{Tabla 1. Diagnóstico diferencial de granulomatosis hepática}

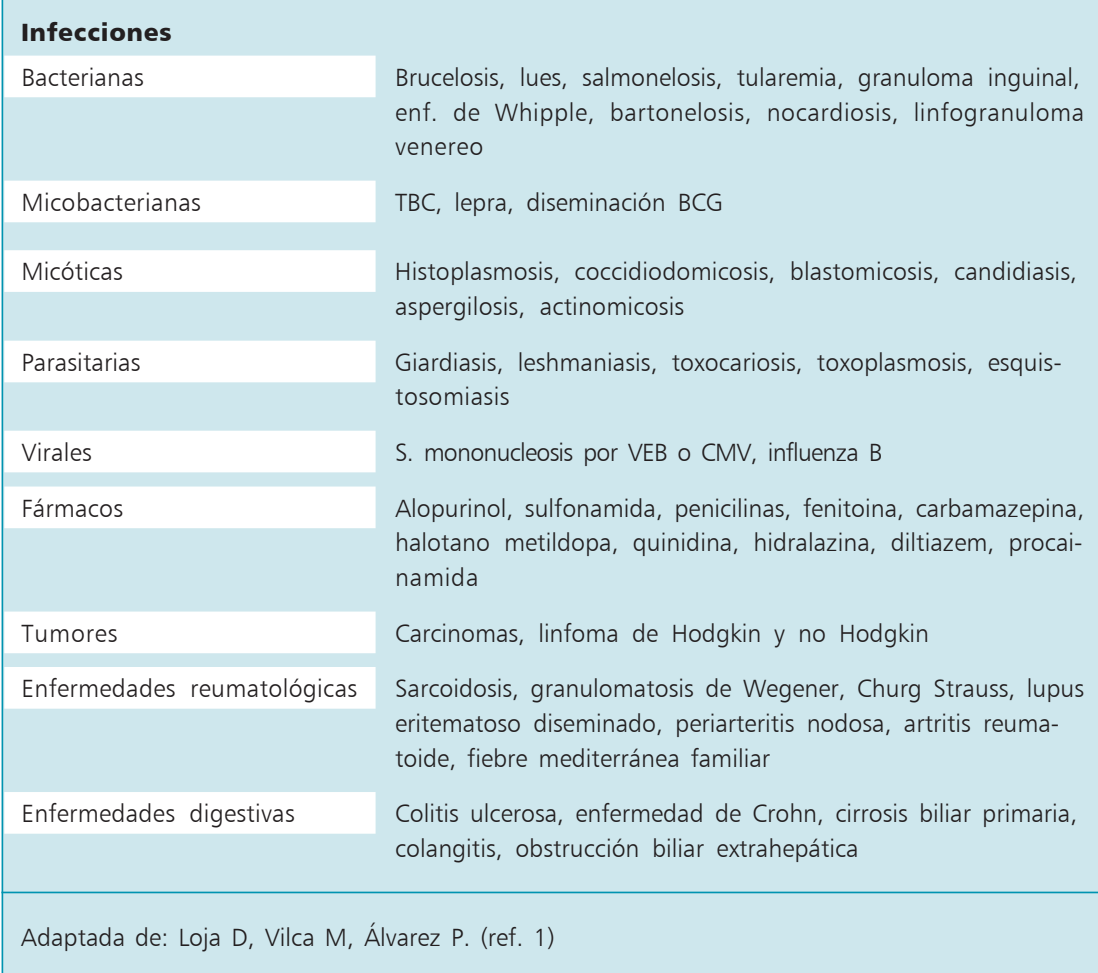




\section{Conclusiones}

La TBC sigue siendo una enfermedad prevalente $\mathrm{t}^{4,9,10}$, que puede presentarse clínicamente de variadas maneras incluido la $\mathrm{FOD}^{1}$, forma en la que se manifestó nuestro caso.

En los pacientes inmunocomprometidos como el presente, las formas extrapulmonares son más habituales que en los inmunocompetentes y la micobacteriosis hepática es una entidad clínica muy poco frecuente, por lo que su diagnóstico requiere de una alta sospecha clínica ${ }^{1,4}$.

Su presentación suele ser inespecífica, siendo más frecuente el hallazgo de fiebre, hepatomegalia dolorosa y colestasia ${ }^{1,6,14}$ síntomas y signos que presentó nuestro enfermo.

La confirmación diagnóstica requiere de métodos invasores como la biopsia hepática, la que en esta ocasión fue determinante en alcanzar el diagnóstico.
Aunque no se pudo obtener muestra para cultivo, la favorable respuesta al tratamiento antituberculoso específico nos hace suponer que se trató de una infección por M. tuberculosis.

\section{Resumen}

Reportamos el caso de un hombre de 42 años, seronegativo para VIH, con fiebre de origen desconocido (FOD), asociada a elevación de transaminasas y fosfatasas alcalinas con patrón colestásico e imágenes hepáticas hipodensas en la tomografía axial computada. La biopsia hepática demostró la presencia de granulomas tuberculosos con visualización de un bacilo con alcohol-ácido resistencia. El cuadro respondió al tratamiento con fármacos antituberculosos presentando caída de curva febril, mejoría del estado general y normalización de parámetros de laboratorio.

\section{Referencias}

1.- Loja D, Vilca M, Álvarez P. Hepatitis granulomatosa tuberculosa como causa de fiebre de origen desconocido. Rev Gastroent Perú 2002; 22: 324-9.

2.- Lewis J, Zimmerman H. Tuberculosis de hígado y vías biliares. En Scholossberg D, ed. Tuberculosis e infecciones por micobacterias no tuberculosas. México. Editorial McGraw-Hill, 2000; 271-299.

3.- Moulding T. Patogenia, fisiopatología e inmunología: orientaciones clínicas. En Scholossberg D, ed. Tuberculosis e infecciones por micobacterias no tuberculosas. México. Editorial McGraw-Hill, 2000; 54-64.

4.- Zúñiga M. Situación de la tuberculosis en Chile-2002 y su posible evolución. Rev Chil Enferm Respir 2003; 19: 179-91.

5.- Havlir D, Barnes P. Tuberculosis in patients with human immunodeficiency virus infection. N Engl J Med 1999; 340: 367-73

6.- Bernabeu R, Paredes P, Latour J, Méndez P,
Lorenzo M, Sánchez F. Absceso hepático tuberculoso en paciente inmigrante con tuberculosis pulmonar. Arch Bronconeumol 2002; 38: 246-8.

7.- Harries A, Maher D, Graham S. TB/HIV. A clinical manual $2^{\text {nd }}$ edition. http://whqlibdoc. who.int/publications/2004/9241546344.pdf

8.- Huang W-T, Wang C-C, Chen W-J, Cheng Y-F, Eng H-L. The nodular form of hepatic tuberculosis: a review with five additional new cases. J Clin Pathol 2003; 56: 835-9.

9.- Farga V. La conquista de la tuberculosis. Rev Chil Enferm Respir 2004; 20: 101-8.

10.- Mackenna M, Mccray E, Onorato I. The epidemiology of tuberculosis among foreignborn persons in the United States, 1986 to 1993. N Engl J Med 1995; 332: 1071-6.

11.- World Health Organization (WHO). Global tuberculosis control-surveillance, planning, financing 2004.

12.- Mahajan S, Sood B R, Thomas M, Thakur S, Singh Pal. Macronodular hepatic tuberculo- sis. J Indian Acad Clin Med 2004; 5: 188-90.

13.- Rufi G, García J F, Solera J, Moreno S. Tuberculosis. En Protocolos clínicos S.E.I.M.C. Aguado J M, Almirante B, Fortún J eds. www.seimc.org/protocolos/ clinicos/proto 7.htm

14.- Fontseré N, Bonet J, Bonal J, Romero R. Tuberculosis hepática en paciente con nefropatía diabética. Una presentación infrecuente. Nefrología 2003; 23: 550-3.

15.- Yorulmaz I, Uncu H, Aker Y, Sakarya A, Isisag A. Hepatic macronodular tuberculosis. Tr. J Med Sciences 1999; 29: 587-9.

16.- Rai S, Thomas WM. Diagnosis of abdominal tuberculosis: the importance of laparoscopy J R Soc Med 2003; 96: 586-8.

17.- Engin G, Acuna B, Acuna B, Tunaci M. Imaging of extrapulmonary tuberculosis. RadioGraphics 2000; 20: 471-88.

18.- Gaya D, Thorburn D, Oien K, Morris A, Stanley A. Hepatic granulomas: a 10 year single centre experience. J Clin Pathol 2003; 56: $850-3$ 